\title{
Migrating In-House Data Center To Private Cloud: A Case STUdy
}

\author{
Majed Alhaisoni \\ College of Computer Science and Engineering, University of Hail \\ Saudi Arabia
}

\begin{abstract}
Internet traffic has become vast in terms of data processing as well as storage which has occurred some limitations on existing traditional infrastructure. Such limitations may include servers, data storage, and computing resources. This has consequently led most of organization to keep investing and expanding their infrastructure in periodical phases. Hence, two factors are always co-related to each other which are TCO (total cost of ownership) and ROT (return on investment) which are critically increased due to the amount of general investment including all expenses as well as human powers. Therefore, a new technology has emerged which is called "Cloud Computing". This terminology has been released recently to work as a supportive to traditional infrastructure which is part of Datacenters. It offers reliability, improved QoS, and efficiency to end-users. This paper presents a novel case study of cloud computing on a large scale environment. Various factors such as TOC and ROI have been taken in consideration on a comparative study with traditional Datacenter. Results have shown considerable improvement on performance, utilization, and investment.
\end{abstract}

\section{KEYWORDS}

Cloud computing, ROT, TCO, QoS, CAPEX, OPEX

\section{INTRODUCTION}

Technology is growing very rapidly towards various directions of innovation including computing and resources. Such development is a key pointer of the massive increase on internet traffic and lack of resources [11] [12] [13]. Therefore, business is moving behind ways of saving computing resources on a low cost effective wise [14]. However, organizations have become more advanced on delivering services and running their infrastructure against some limitations of budget and cost. To narrow down the subject, educational sector is one of the largest sectors that seek state-of-art solutions on building IT infrastructure, with maximum low cost effect.

In educational sector, there are many of the running systems on different levels such Students Information System (SIS) ,HR system, ERP (Enterprise Resource Planning), and eLearning systems. These systems acquired higher demand on bandwidth and computing resources, and most importantly require high availability. Legacy infrastructure has shown some limitations as shall be shown on the rest of this paper. Systems upgrades of software and hardware are imperative items on such organizations' resource meetings and it is always be a pressure on the budgets. This situation is likely to be became worse in nowadays financial crisis.

Cloud computing has emerged as a new technology on late 2007 [1] [15] [16] and since then it has shown to be an upcoming topic of research owing to the ability of granting dynamic infrastructure on various levels such as services and computing resources. Different paradigms have shown to present such technology such as grid computing, cluster computing and eventually 
cloud computing [17] [18]. More emphasis has been given to the latter which is "Cloud" [19]. It has given more potentials to the owner of business and normal users to access their needs at anytime, anywhere based upon their demand without running their own infrastructure [20]. Certainly, cloud computing has proven to be attractive to academic institution [21]. Universities are always looking to upgrade their software and IT hardware in order to meet students and staff requirements with the rapid developments in digital technologies. Cloud computing is an option to achieve such motivations with budget affordability [23].

Over the last few years, many companies have used clouds to build highly scalable systems. However, cloud computing is not just for start-ups; enterprises are attracted to cloud-based services as cloud providers market their services as being superior to in-house datacentres in terms of financial and technical dimensions e.g. more cost effective, equally or perhaps more reliable, and highly scalable.[1,2,22].

This paper presents a novel case study of how cloud computing could be an optimum solution for educations, and essential to note that which cloud solution is better is out of the scope of this case study. Moreover, Migration of University of Hail Data centre to private cloud and potential benefits of private cloud computing concept which become evident: better control, access, security and efficiency than today's data centre with the agility required for business innovation at substantially lower costs from virtualization is also presented.

\section{RELATED WORK}

Cloud computing has been shown recently on research areas, so not much studies have been carried out on this new trend of technology in terms of case studies. However, cloud computing as a technology has been looked at from different angles. For instance in [7], the authors define cloud services and offer insight into architecture and how it could be leveraged for virtualization. Moreover, authors have given insight into on market-based resource management strategies that embrace both customer-driven service management and computational risk management to sustain Service Level Agreement (SLA)-oriented resource allocation.

Authors in [8] has given more emphasis on concept of cloud computing and some of the issues that cloud could address as new technology. Also it has given an idea of what research topics would be considered on implementation of cloud today.

However, cloud establishment has been considered on [9] as several general examples of cloud users were provided and a case study of the University of Westminster was presented in details. It was shown that educational establishments are most likely ready to take over cloud computing due to funding issues and economic crisis.

Cloud computing deployment in educational sector has been surveyed by [10] and in particular at university of California (UC) at Berkeley. It was shown that cloud is very handful to use on their teaching materials where all courses were migrated into cloud instead of being stored locally.

In contrast to abovementioned studies, this paper presents a novel case study looking at performance of real cloud experience at educational sector. Furthermore, a comparative study with aging old traditional data centre is presented. Considerable factors have been examined on both environment to gauge cloud performance and advantages over traditional servers and storages. 


\section{CASE STUDY}

In this section, background information of the case study environment shall be covered in terms of selected institute; also various factors such as challenges and problems encountered on old environment are highlighted and compared to the new paradigm of data centre as follow:

\subsection{University of Ha'il}

University of Hail is a Governmental, non-profit university serving around 40,000 students in the province of Hail, at north of Saudi Arabia. UoH was officially established by Royal Decree in 2005. It reflects the development of the National Talent and Creativity by providing 21st Century Learning Competencies, Linking education to social and economic, preparing professionals to be ready to overcome current and future challenges.

UOH has faced rapid growth in its community in the last 5 years. 2000 students were enrolled in the first batch. Since that time, the University enrollment has grown to a level that is expected to exceed 50,000 by the 2015-2016 academic year.

It has 11 campuses within Hail city as well as in remote sites around at the distance from minimum 5KM to maximum $300 \mathrm{KM}$. UoH offers undergraduate degrees in 14 colleges such as but not limited to Sciences, Engineering, Medical and Arts Majors. Also, It serves postgraduate courses on various subjects.

\subsection{Challenges}

Various challenges are arising due to the nature of services that are delivered to the customers. As most of services are consumed on daily basis if it's not hourly which bring a load on IT management to deal with such rapid demand. Following are shortlist of some of the challenges which are faced frequently as follow:

\subsubsection{Emerging Business Requirements}

In recent years, in response to the massive growth in university community (faculty and students) population and under E-Government initiative, the UoH has many successful implementation of Eservices and advanced information Systems (SIS, ERP, ECM, BPM) to achieve excellence in research and academics, as well as higher administrative efficiency. However, this has put a lot of pressure on old $\mathrm{UoH}$ data centre to cope with such rapid transformation and had been failing behind due to having aging physical infrastructure.

\subsubsection{Aging and Accidental Infrastructure}

Old data Centre comprised of 60+ physical servers, with an average age of six years (some up to 8 -years-old). Most of these servers are facing hardware reliability and had reached capacity. Disaster recovery capabilities were very limited, relying on old fashion for backup. Furthermore, in case of server failure, then another had to be procured and physically deployed in its place. This consequently would require time and cost where services cannot be interrupted at all due to having various research experimental labs ongoing as well as other critical departments working full-time. Moreover, Recovering data and applications were difficult and time-consuming exercises. 


\subsubsection{Management and Operational Complexity}

Traditional data centre requires intensive management and maintenance; hence, university's IT staff time and efforts mostly spend on troubleshooting and keeping the services up and running as shown in figure 1. IT was extremely short of the resources to expand the infrastructure rapidly enough to meet the SLAs and community's high expectations. Furthermore, such legacy environment is a key cause of not getting more innovation and creativity from IT staff due the fact of always being busy on managing and monitoring whole infrastructure.

\subsubsection{Total Cost of Ownership (TCO)}

Maintaining these diverse technologies became difficult and costly. Acquiring together new technologies on our own was extremely risky, complex and time consuming. Any iterations or additional nodes were always costly across levels. Additionally, single point of failure was a critical issue for legacy data Centre due to the fact of not placing many physical servers due to budget constraints and cost.

Therefore, the university was in need of migrating to a solution that offer rich virtualization and cloud capabilities while freeing our administrators to focus more on innovation.

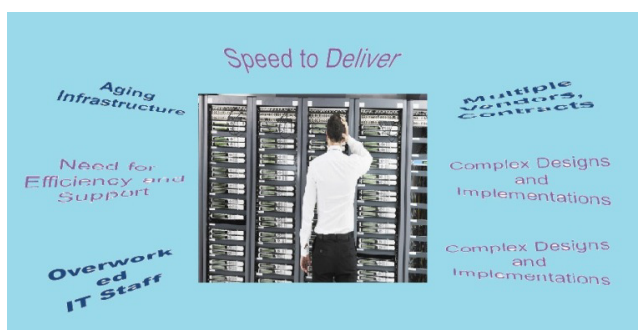

Figure 1 Challenges in Datacentre

\subsection{CLOUD BASED SOLUTION}

Could computing has been offered on various models by different vendors. Each and every vendor is looking at cloud from certain viewpoint which gives more innovation and new trend. Hence, in order to adapt such technology, it was essential to survey as many as possible offered solution on cloud-based, taking into consideration university demand and budget limitation. However, after studying the existing architecture and the business requirements, it is found that there are solutions available for public and private cloud as shown in below in figure 2 .

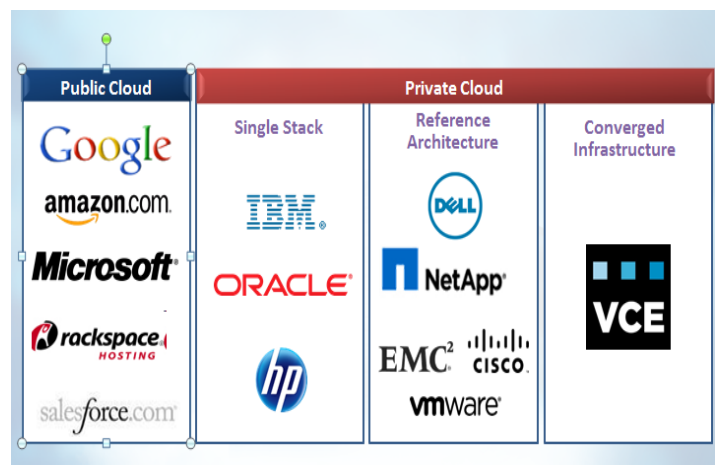

Figure 2 Some of the available solutions 
After studying available solutions, it was evident to the IT management that most of available solutions are capable to handle university needs and demand. However, since the aim of this paper is to show how cloud perform over traditional servers and infrastructure. On the other hand, answering the question of which solution is better is out of the scope of this case study. Therefore, university has selected VCE solution which is called VBlock. UoH has deployed a Vblock System 720 which is pre-tested, pre-engineered, and pre-configured. Within 4 days of powering up the Vblock System, applications were running. The entire migration was completed in four weeks. The Vblock System now provides the performance, reliability, agility and simplified management needed to support UOH's expansion. Applications running on the Vblock System are fully virtualized.

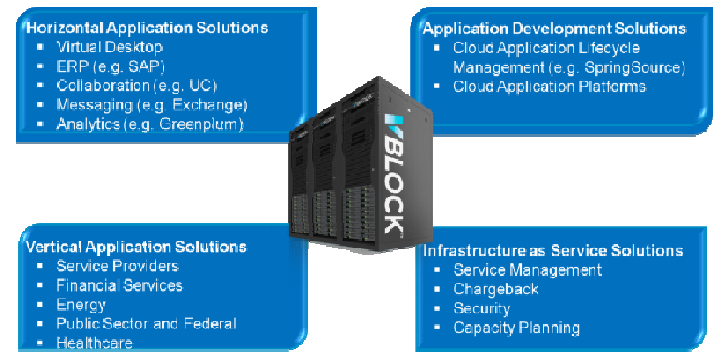

Figure 3 Vblock Solution [4]

The Vblock System has provided the performance, reliability, agility and simplified management needed to support UOH's expansion. The infrastructure hosts MS SQL Server, Oracle RAC, MS SharePoint, MS Exchange and Core business applications Academic SIS, Oracle ERP and .Net based E-Services. The applications are all running on the Vblock System are fully virtualized. Figure 4 shows how the selected solution is structured and joint together as one component. It clearly shows how private cloud could be built and fully virtualized, not only this but pool of resources are all managed from a single console.

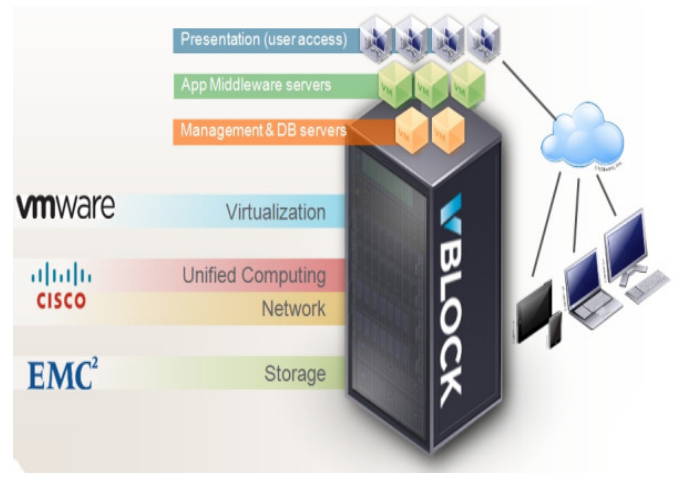

Figure 4 Vblock technology [4]

\section{PERFORMANCE EVALUATION AND RESULTS}

In order to compare between the new deployed infrastructure on cloud platforms versus tradition infrastructure, experimental work has been conducted on two different datacentre at university of Ha'il. However, it is essential to convey that environment have been similarly run on two different sites with similarities on applications and systems deployed on both sites. Therefore, in order to gauge and validate the performance of both trends, various factors have been considered which are shown on the following: 


\subsection{Smaller Footprint}

Private Cloud implementation sped deployments, increased IT productivity, enhanced agility, saved capex and opex, and enabled staff to spend more time for R\&D, and achieve user satisfaction. IT is now a key enabler of UOH's growth and educational objectives. Small foot print to save energy, space Consolidated server equipment racks down from 13 to 6, Physical servers from 65 server to 24 , so hardware occupies 50 percent less resources in the data centre.

Figure 5 gives a clear insight between traditional datacentre (a.k.a pre-cloud) and Cloud based solution. It has been noticed that ac clear reduction in acquisition/maintenance expenditures on hardware (servers, storage, network, etc.) and software are saved by $40 \%$, servers by $63 \%$, and power by $50 \%$; not only that but also It has reduced facilities space requirements by $53 \%$. Therefore, such valuable diversification among two paradigms would surely encourage public and private sectors both to migrate and join the cloud era.

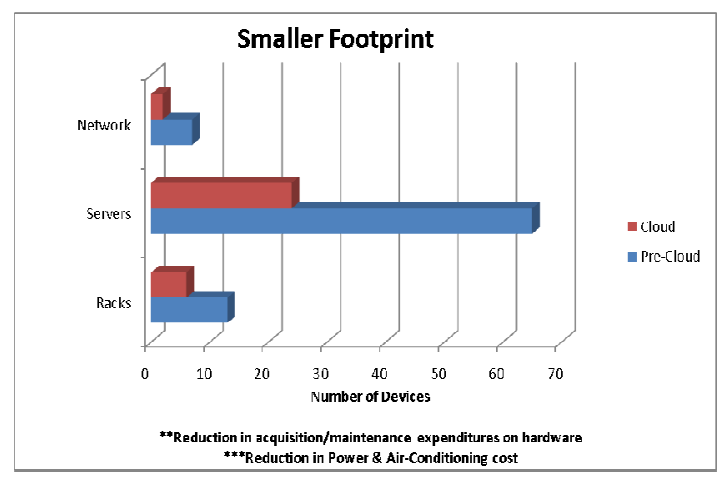

Figure 5 Smaller Footprint

\subsection{Predictable Performance}

Performance is a key factor on IT environment; hence, systems are always gauged by their capability and scalability on performing and responding very prompt on end users or business requirements. Thus, in order to achieve high performance with high availability, cloud solution has been recently a trend to accelerate and boost up processes on IT environment. On this case study, It has been found out and investigated that virtualisation is taking place on the new environment up to 100 percent of UOH Tier 1 applications which maintains high service levels for roughly 40,000 users. Therefore, the Private cloud has shown easily critical workloads, the resources to get the required response times, especially for critical applications such registration, payroll, and other demanding systems. Not only this but also database lookups now get millisecond responses. Furthermore, Private cloud has proven to keep running without downtime, at incident of any hardware failure, this is backed-up on a virtual server replication which offers high availability up to 99.99. Figure 6 shows a comparative measurement between pre-cloud scenario and cloud paradigm. It is very obvious that cloud (which is adapting Vblock solution) has proven very clear reduction in terms of server incidents due to redundancy offered by virtualisation. Furthermore, time cost for fixing or returning any single server back to normal is consuming very long time whereas on cloud solution is easily figured out from a single console management system. 


\begin{tabular}{|l|r|r|}
\hline Downtime Measures & Pre-Vblock Platform & Vblock Platform \\
\hline Server incidents & 12 & 1 \\
\hline Hours needed to fix the problem & 5.2 & 1.8 \\
\hline Server downtime hours & 70.6 & 0.8 \\
\hline Downtime hours per user & 10 & 0.1 \\
\hline & & \\
\hline $\begin{array}{l}\text { Note: All downtime and incident } \\
\text { measures arefor an entire year. }\end{array}$ & & \\
\hline
\end{tabular}

Figure 6 Downtime Measurement

It is also worth mentioning that predictable performance has also enabled UOH to virtualize key databases with confidence, including SIS, Oracle ERP, ECM databases, which are accessed by its customers on a $24 \times 7$ basis. Use of Storage Virtualization provisioning technology FAST VP and thin-provisioning improved resource utilization and increase performance.

\subsection{IT efficiency}

The private cloud platform provides $\mathrm{UOH}$ to manage everything from a single console. UOH estimates the division's three-member IT team regained 60 to 70 percent of the time spent managing their older systems. With the efficiency gains enabled by an integrated, virtualized infrastructure and consolidated support model, UOH IT has significantly more time to focus on innovative projects to promote the university's long-term vision for Academic excellence.

Growing from 4 to 90 terabytes business data with no increase in IT Staff and freeing more time for IT to focus on delivering solutions instead of troubleshooting.

New Sever provisioning is $20 \mathrm{x}$ time faster than was on legacy infrastructure. New virtual servers can be provisioned in round 15-20 minutes, compared to several hours or even days using the previous physical infrastructure.

Figure 7 depicts estimated cost the two scenarios (pre-cloud and cloud), It is obviously shown that cloud based solution is in a position to save a lot of cost for any organisation if they plan migration to cloud very carefully.

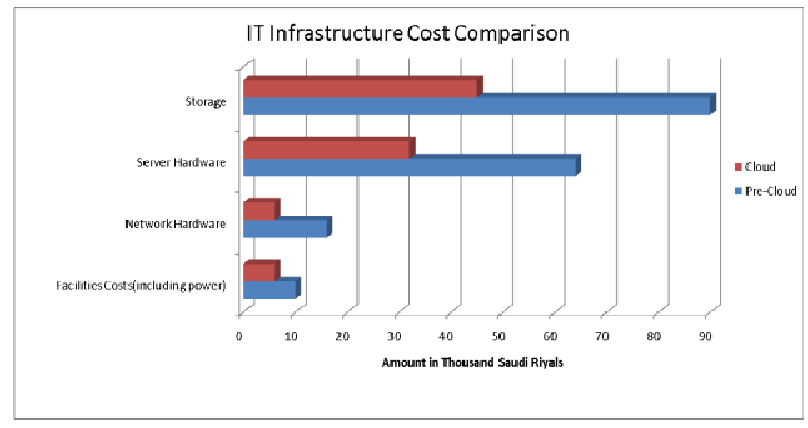

Figure 7 : IT Infrastructure Cost Comparison

\subsection{Operation and maintenance}

This section gives another insight of various evidences have been figured out by the conducted study towards cloud trend in terms of different factors. Below figures are Comparing old vs. 
Cloud based solution's Operating Saving with respect to operation cost, management saving, operation saving, investment and saving flow and finally Total Cost of Ownership is considered.

By simplifying operations, the Cloud based solution provides significant cost savings across different data centre operating cost categories over the five year period as indicate in figures 8 and 9 below. Furthermore, cloud solution implies an early capital outlay, the benefits over time add up as indicated by figure 11 below. Overall, It is apparent that the benefits of private cloud computing concept have become evident: better control, access, security and efficiency than today's data centre with the agility required for business innovation at substantially lower costs from virtualization as shown in figure 10 and 12. Therefore, If any sort of business requires multiple IT services, no longer needs to assemble your own hosting/support/provisioning, management tools, or planning equipment for future growth, cloud has shown efficient performance over traditional legacy heterogeneous servers.

\begin{tabular}{|c|c|c|c|c|}
\hline Hard Costs & old & Cloud & Savings & \%Reduction \\
\hline HWISW Lease Payments & $\$ 0$ & $\$ 0$ & $\$ 0$ & 00 \\
\hline HWISW Maintenance & $\$ 281,325$ & $\$ 0$ & $\$ 281,325$ & 1009 \\
\hline Power and Cooling & $\$ 5,108,963$ & $\$ 997,039$ & $\$ 4,111,925$ & $80^{\circ}$ \\
\hline Floor Space & $\$ 639,637$ & $\$ 802,682$ & $\$ 163,045$ & .250 \\
\hline Management Costs & $\$ 4,412,973$ & $\$ 4,080,804$ & $\$ 332,169$ & 80 \\
\hline Downtime & $\$ 19,892,273$ & $\$ 1,989,227$ & $\$ 17,903,045$ & 900 \\
\hline Total Operating Costs & $\$ 30,335,171$ & $\$ 7,869,752$ & $\$ 22,465,419$ & $74^{\circ}$ \\
\hline
\end{tabular}

Figure 8 Operating cost saving Comparison

\begin{tabular}{|c|c|c|c|c|}
\hline 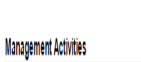 & old & Clovd & $\operatorname{lig} \mid$ | & Merouction \\
\hline 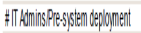 & 9.4 & 23 & 72 & 760 \\
\hline 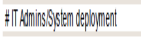 & I. & 66 & 28 & 3W \\
\hline 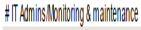 & 126 & 88 & 38 & 30 \\
\hline Cherl Oertard & 25 & 323 & 98 & 4th \\
\hline 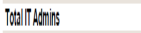 & 54. & 300 & 40 & 77 \\
\hline llangenenert Costs & 4.412973 & \$40000004 & w210 & 8 \\
\hline
\end{tabular}

Figure 9 Management Saving

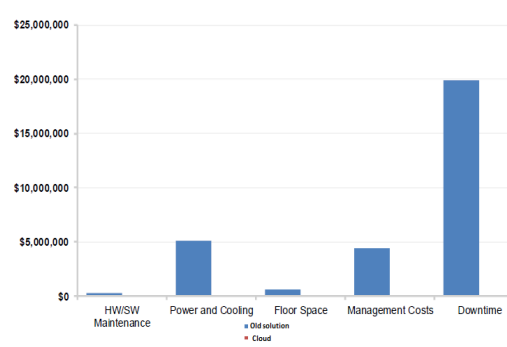

Figure 10 Operation Saving

\begin{tabular}{|c|c|c|c|c|}
\hline Hard Costs & old & Cloud & Savings & \%Reduction \\
\hline HWISW Investment & $\$ 1,696,515$ & $\$ 3,935,805$ & $\$ 2,239,290$ & .132 \\
\hline Vblocks & $\$ 0$ & $\$ 3,935,805$ & $\$ 3,935,805$ & 0 \\
\hline Sevvers & $\$ 782.532$ & so & $\$ 782.532$ & $100 \%$ \\
\hline Servers - Racks \& Chassis & $\$ 71,680$ & $\$ 0$ & $\$ 71,680$ & 1009 \\
\hline VM Licenses & $\$ 0$ & so & $\$ 0$ & $0 ?$ \\
\hline Switches & $\$ 700,000$ & so & $\$ 700,000$ & $100^{\circ}$ \\
\hline Syitches - Racks \& Cabling & $\$ 0$ & so & $\$ 0$ & $0 ?$ \\
\hline Storage & $\$ 142,302$ & so & $\$ 142,302$ & $100 \%$ \\
\hline Addtitional Investment Caotal & $\$ 0$ & so & $\$ 0$ & $0 ?$ \\
\hline$\overline{\text { Total Capital Costs }}$ & $\$ 1,696,515$ & $\$ 3,935,805$ & $\$ 22,239,290$ & .1329 \\
\hline
\end{tabular}

Figure 11 Investment and saving flow

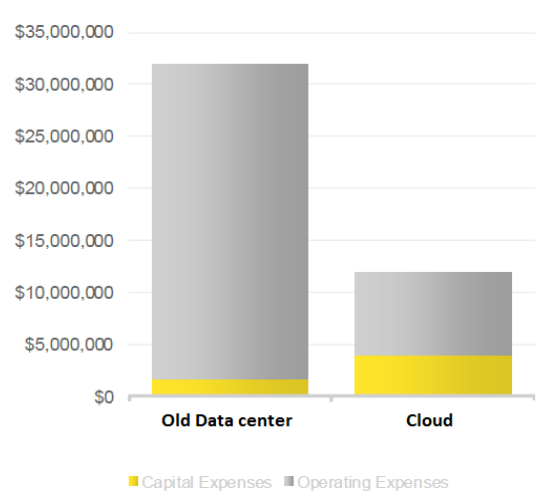

Figure 12 : TCO Comparison 


\section{CONCLUSION AND FUTURE WORK}

This paper has contributed to the fields of cloud area by pure raw case study comparing and testing the performance of cloud over traditional servers. Cloud computing is a disruptive technology that is set to change how IT systems are deployed because of its apparently simple and scalable nature. The findings of this case study show that cloud computing can be a significantly cheaper alternative to purchasing and maintaining system infrastructure in-house. Furthermore, cloud computing could potentially eliminate many support-related issues since there would be no physical infrastructure to maintain. Despite these advantages, this case study has showed that there are important socio-technical issues that need to be considered before organizations could migrate their IT systems to the cloud. Finally, the system infrastructure in the case study has evidently shown Operating Costs $74 \%$ over 5 Years, $8 \%$ saving on management , and $63 \%$ on cash purchase.

\section{REFERENCES}

[1] M. Armbrust, A. Fox, R. Griffith, A. Joseph, R. Katz, A. Konwinski, G. Lee, D. Patterson, A. Rabkin, I. Stoica, and M. Zaharia, "Above the Clouds: A Berkeley View of Cloud omputing.," 2009.

[2] H. Erdogmus, "Cloud Computing: Does Nirvana Hide behind the Nebula?," IEEE Software, vol. 26, 2009, pp. 4- 6.

[3] M.A. Vouk, "Cloud computing issues, research and implementations," 30th International Conference on Information Technology Interfaces (ITI 2008), Cavtat/Dubrovnik, Croatia: 2008, pp. 31-40.

[4] The 5 Major Themes of EMC World 2013, Andrew Rice on Thu, May 16, 2013

[5] I. Sriram and A. Khajeh-Hosseini, "Research Agenda in Cloud Technologies," Submitted to 1st ACM Symposium on Cloud Computing (SOCC 2010), 2010.

[6] A. Khajeh-Hosseini, I. Sommerville, and I. Sriram, "Research Challenges for Enterprise Cloud Computing," Submitted to 1st ACM Symposium on Cloud Computing (SOCC 2010), 2010.

[7] Rajkumar Buyya et al "Cloud computing and emerging IT platforms: Vision, hype, and reality for delivering computing as the 5th utility", Future Generation Computer Systems 25 (2009), pp. 599_616.

[8] Mladen A. Vouk, "Cloud Computing - Issues, Research and Implementations", Journal of Computing and Information Technology - CIT 16, 2008, 4, pp. 235-246

[9] Nabil Sultan, "Cloud computing forb education: A new dawn?", International Journal of Information Management 30 (2010), pp. 109-116

[10] Fox, A. (2009). Cloud computing in education. Berkeley iNews, https://inews.berkeley.edu/articles/Spring2009/cloud-computing, (accessed on: 20 April 2015).

[11] Ahmed Mohamed Gamaleldin , "An Introduction to Cloud Computing Concepts" , Software Engineering Competence Center 2013 ,page 2.

[12] Nariman Mirzaei , Cloud Computing, fall 2008 ,page 2 to 4.

[13] Jangareddy gudem, Vijayawada "Cloud computing : an overview" ,page 2-4

[14] Santosh Bulusu ,Kalyan Sudia “A Study on Cloud Computing”, 012012 ,Page 16.

[15] Mike P. Papazoglou, "Service -Oriented Computing: Concepts, Characteristics and Directions", Tilburg,University, INFOLAB ,page 1.

[16] Amit Singh, “An Introduction to Virtualization”,January 2014, page 1.

[17] The Cloud , thecloudtutorial.com Tutorial ,page 1.

[18] Echorack systems , "Cloud computing Types”,Page 1.

[19] The appcore Blog, "3 Types of Cloud Service Models", page 1.

[20] Clinton D Souza "Security concern with SaaS layer of Cloud computing", 01/29/2013 ,page 5.

[21] Lord Crusader "Problems faced by cloud computing", page 2- 4

[22] U.S. National Institute of Standards and Technology "Fact Sheet" .page 3-4.

[23] Mathur, P; Nishchal, N.; (2010), "Cloud Computing: New challenge to the entire computer industry", 2010 1st International Conference on Parallel, Distributed and Grid Computing (PDGC - 2010), pp 223. 